\title{
Niveles de grano de soya integral tostado en dietas para codornices japonesas (Coturnix coturnix japonica) en fase inicial de postura
}

\author{
Levels of soybean grain toast in diets for Japanese quail (Coturnix coturnix \\ japonica) in initial phase of stance \\ Marín V. Indira ${ }^{1}$, Enciso P. Marelis ${ }^{1}$ y Hurtado Nery V. L. ${ }^{2}$ \\ ${ }^{1}$ Medicos Veterinarios Zootecnistas \\ ${ }^{2} \mathrm{MVZ}$. PhD, Docente Universidad de los Llanos \\ vhurtado@unillanos.edu.co
}

Recibido 02 Agosto 2012, Aprobado 17 Octubre 2012

\section{RESUMEN}

Este trabajo fue realizado con el objetivo de evaluar el efecto de la inclusión de grano de soya integral tostado en la ración para codornices japonesas en la fase inicial de postura. Fueron utilizadas 250 aves distribuidas en un diseño experimental completamente al azar, con cinco tratamientos, cinco repeticiones y 10 aves por repetición. Los tratamientos estaban constituidos por cinco raciones con $0,5,10,15$ y $20 \%$ de inclusión de grano de soya integral tostado. La fase experimental tuvo una duración de 84 días. Los resultados obtenidos fueron: Producción de huevos 60, 58, 54, 57 y 54\%; peso del huevo 10.5, 10.7, 10.5, 10.4 y 10.4; consumo diario de ración 24.2, 23.8, 23.9, 23.0 y 24.9; Conversión alimenticia ( $\mathrm{kg}$ de ración $/ \mathrm{kg}$ de huevo 3.84, 3.83, 4.21, 3.88, 4,43; $\mathrm{kg}$ de ración/docena de huevos $0.484,0.492,0.531,0.484$ y 0.453 ; grosor de la cáscara $0.17,0.16,0.17,0.18$ y 0.17 ; peso específico $1.072,1.071,1.073,1.070$ y 1.071 ; Unidades Haugh 93.11, 91.37, 93.25, 91.73 y 92.28 respectivamente para los niveles de inclusión de $0,5,10,15$ y $20 \%$ de grano de soya integral tostado respectivamente. Los resultados no arrojaron diferencias significativas entre los tratamientos para las variables zootécnicas ni para las características del huevo. En conclusión, el grano de soya puede ser incluido hasta en $20 \%$ en raciones para codornices japonesas en la fase inicial de postura. 
Palabras claves: Alimentos alternativos, codornices japonesas, conversión alimenticia, grano de soya.

\begin{abstract}
A research was conducted with the objective to evaluate different levels of toasted whole soybean on eggs production and egg quality on an initial laying phase. It was a total 250 birds, used in completely randomized experimental design with five treatments and five replicates and ten birds for repetition. The treatments were five diets with $0,5,10,15$ and $20 \%$ of grain soybean whole toasted. The experimental phase was of 84 days. The results were egg production 60, 58, 54, 57 y 54\%; egg weight 10.5, 10.7, 10.5, 10.4 and 10.4; feed intake daily 24.2, 23.8, 23.9, 23.0 and 24.9; feed conversion (kg diet/kg egg 3.84, 3.83, 4.21, 3.88 and 4,43; $\mathrm{kg}$ diet/dozen eggs $0.484,0.492,0.531,0.484$ and 0.453 ; shell thickness $0.17,0.16,0.17,0.18$ and 0.17 ; specific gravity 1.072, 1.071, 1.073, 1.070 and 1.071; Haugh units 93.11, 91.37, 93.25, 91.73 and 92.28 for de $0,5,10,15$ and $20 \%$ of grain soybean whole toasted. The results showed no significant differences between treatments for production characteristics and eggshell quality. Results obtained indicate that toasted whole soybean can be including in initial laying diets of Japanese quails up to $20 \%$.
\end{abstract}

Keywords: Alternative foods, feed conversion, japanese quails, soybean grain.

\title{
INTRODUCCIÓN
}

Las codornices son aves de gran precocidad y alto rendimiento en la producción de huevos, la producción de huevos de codornices es una actividad que ha crecido en los últimos años. Según Vásquez y Ballesteros, (2007) en Colombia existe una población aproximada de 800.000 codornices. El bajo consumo diario de alimento de estas aves, permite que el productor pueda elaborar, almacenar y manejar el concentrado en la misma granja, el alimento debe ser formulado para atender los requerimientos nutricionales de las codornices en postura. 
El grano integral de soya es un alimento alternativo con alto contenido proteico, de limitado uso por la presencia de factores antinutricionales (Barreto et al., 2010). Para obtener el máximo aprovechamiento del grano integral de soya, es necesario someterlo a un proceso térmico adecuado que permita inhibir la actividad de los factores antinutricionales. Dichos metabolitos son termolábiles y la destrucción del efecto de estos principios depende de la intensidad de la temperatura y el tiempo de duración del proceso. Por la baja temperatura o el poco tiempo de procesamiento los principios antinutricionales continúan activos, al contrario, el procesamiento exagerado determina una destrucción irreversible de aminoácidos como la lisina afectando severamente la calidad de la proteína.

Los principales métodos utilizados para el procesamiento de la soya integral son: tostado por vapor húmedo, tostado por vapor seco, tostado por aire caliente, micronización, microondas, extrusión en seco y extrusión húmeda (Butolo, 2002). De todas formas, para tener completa confianza en el procesamiento de la soya es necesario realizar un buen control de calidad y de análisis nutricionales.

El control de calidad de materias primas debe incluir la evaluación del contenido de factores antinutricionales y disponibilidad de proteína y aminoácidos, para lo cual se debe determinar el índice de actividad de ureasa e índice de inhibición de tripsina. En condiciones prácticas, se recomienda hacer una evaluación para inhibidores de tripsina, basados en el índice de ureasa que en el grano de soya crudo está entre 2.0-3.0 y el grano procesado en óptimas condiciones se encuentra entre 0.02 a 0.05 . El índice inhibidor de tripsina en el grano crudo está presente en un 75 a $80 \%$ y en el grano procesado está por debajo de $0.10 \%$. El índice de biodisponibilidad de lisina genera información de gran utilidad para evaluar el valor nutricional de los alimentos.

En la producción comercial de soya se obtiene grano integral, torta de soya y aceite, materiales que se caracterizan por ofrecer valores óptimos en nutrientes para aves y cerdos como fuente de proteína y energía (Rostagno, 2005). Se ha 
considerado que la torta de soya como fuente vegetal, ofrece la mayor calidad proteica $(46 \%)$ y el mejor equilibrio en aminoácidos esenciales con un alto porcentaje de digestibilidad (82\%).

El objetivo de este trabajo fue evaluar el efecto de diferentes niveles de inclusión de grano de soya integral tostada, SIT, sobre los parámetros productivos y algunas características de calidad del huevo de codornices japonesas en la fase inicial de postura.

\section{MATERIALES Y MÉTODOS}

El trabajo fue realizado en la Universidad de los Llanos, localizada a $12 \mathrm{Km}$. de la Ciudad de Villavicencio, Meta, ubicada a 420 metros sobre el nivel del mar, temperatura promedio anual de $25^{\circ} \mathrm{C}$, precipitación fluvial de $4050 \mathrm{~mm}$ y humedad relativa del 75\% (IDEAM, 2002).

Las aves fueron distribuidas en un diseño experimental totalmente al azar, con cinco tratamientos, cinco repeticiones y 10 aves por repetición, para un total de 250 aves. Los tratamientos fueron constituidos por los niveles de inclusión de 0, 5, 10,15 y $20 \%$ de harina de grano de soya integral tostada. Las dietas fueron isoenergéticas e isoprotéicas formuladas para atender los requerimientos nutricionales en la fase inicial de postura (NRC, 1994), las raciones contenían además harina de arroz, torta de soya y maíz amarillo molido, vitaminas y minerales (Tabla 1). Las codornices fueron alojadas en jaulas de $1.4 \times 1.0 \times 0.30$ $\mathrm{m}$, en baterías modulares de cinco pisos y tres divisiones por piso, acondicionadas con bebederos automáticos y comederos lineales (Figura 1). Las aves fueron recibidas de 30 días de nacidas, la fase experimental, tuvo una duración de 84 días.

Previamente el grano de soya fue sometido a tratamiento térmico, el cual consistía en tostado en seco a $90^{\circ} \mathrm{C}$ por 5 minutos, posteriormente se pasaba por un molino eléctrico de martillo. 
Tabla 1. Composición de las raciones experimentales

\begin{tabular}{lrrrrr}
\hline \multirow{2}{*}{ Ingrediente, \% } & \multicolumn{6}{c}{ Niveles de grano soya integral tostado, \% } \\
& \multicolumn{1}{c}{$\mathbf{0}$} & \multicolumn{1}{c}{$\mathbf{5}$} & \multicolumn{1}{c}{$\mathbf{1 0}$} & \multicolumn{1}{c}{$\mathbf{1 5}$} & \multicolumn{1}{c}{$\mathbf{2 0}$} \\
\hline Aceite de soya & 4.015 & 2.920 & 1.825 & 0.729 & 0.000 \\
Carbonato de calcio & 5.581 & 5.583 & 5.589 & 5.988 & 6.985 \\
Harina de arroz & 15.000 & 15.000 & 15.000 & 15.000 & 15.000 \\
Maíz & 42.076 & 42.516 & 43.397 & 42.888 & 36.257 \\
Harina de carne & 3.000 & 3.000 & 3.000 & 3.000 & 3.000 \\
Torta de soya & 29.485 & 25.139 & 20.792 & 16.446 & 12.283 \\
Grano de soya integral & 0.000 & 5.000 & 10.000 & 15.000 & 20.000 \\
tostado & & & & & \\
Premezcla vitaminas ${ }^{1}$ & 0.200 & 0.200 & 0.200 & 0.200 & 0.200 \\
Metionina & 0.142 & 0.141 & 0.141 & 0.141 & 0.141 \\
Sal & 0.500 & 0.500 & 0.500 & 0.500 & 0.500 \\
Análisis calculado & & & & & \\
Proteína bruta, \% & 20.00 & 20.00 & 20.00 & 20.00 & 20.00 \\
EM, Kcal/kg & 2900 & 2900 & 2900 & 2900 & 2900 \\
Fósforo disponible & 0.35 & 0.35 & 0.35 & 0.35 & 0.35 \\
Calcio, \% & 2.50 & 2.50 & 2.50 & 2.50 & 2.50 \\
Lisina, \% & 1.00 & 1.00 & 1.00 & 1.00 & 1.00 \\
Metionina, \% & 0.45 & 0.45 & 0.45 & 0.45 & 0.45 \\
\hline
\end{tabular}

${ }^{1}$ Lutavit, contiene por Kg: Vit. A, 20.000.000 UI; Vit D3, 6.000.000, UI; Vit, E, 16.000, UI; Vit K (menadiona), 6.000, mg; Vit B1 (tiamina), $1.000 \mathrm{mg}$; Vit. B2 (riboflavina) 9.000, mg; Vit B6 (piridoxina, 1.000, mg; Vit. B12 (cianocobatamina), 24, mg; Ácido pantoténico, 12.000 mg; Niacina, 12.000 mg; Vit. H (biotina), 40 mg; Ácido fólico, 400 mg; Antioxidante, 50.000 mg.

Determinación de la calidad del huevo. Los análisis para determinar la calidad del huevo fueron realizados en el Laboratorio de Nutrición Animal de la Universidad de los Llanos, se tomaron al azar dos huevos por réplica cada seis semanas. Se realizó la prueba de gravedad específica, utilizando 3 cubos de 
plástico de 4 litros de capacidad, sal sin yodo, 1 picnómetro de $20 \mathrm{ml}$, coladores de plástico para sumergir los huevos dentro de las soluciones salinas, bandeja para los huevos para cada nivel de peso específico. Se prepararon cuatro soluciones que proporcionaron los pesos específicos 1.070; 1.070-1.075; 1.075-1.080 y mayor de 1.080. Estas soluciones se prepararon con 454 gramos de sal por 3.7 litros de agua. Una vez realizadas las soluciones se comprobó la densidad con la técnica picnométrica, calculando la densidad del agua y gravedad específica de cada una de las soluciones. Comprobada la densidad, se sumergieron los huevos en las distintas soluciones, comenzando con la de más bajo peso específico y continuando hasta la más alta. La solución de más alta concentración en que flotan los huevos, corresponde a la gravedad específica de los mismos. El grosor de la cáscara se midió con un tornillo micrométrico 0 - 25 milímetros con precisión 0.01 , en tres muestras de los polos ancho, angosto y ecuatorial de cada huevo, estableciendo posteriormente el promedio respectivo. Para medir las Unidades Haugh, Sobre una superficie plana (caja de petri) se rompió el huevo, sin destruir el albumen denso. La medición se realizó con un calibrador Pie de Rey, de 0,02 $\mathrm{mm}$ de precisión y a una distancia de 7.8 milímetros aproximadamente del borde de la yema sobre la parte plana del albumen denso. Para hacer la conversión a unidades Haugh se aplicó la ecuación descrita por Wineland, (1988).

Unidades Haugh $=100 \log \pitchfork \llbracket\left(h-1,70^{*} p+7.6 \rrbracket\right)$.

$100 \log (\mathrm{h}-1.70 \times \mathrm{p}+7.6)$

Donde:

$\mathrm{H}=$ altura del albumen denso

$\mathrm{P}=$ peso del huevo

Las aves fueron alimentadas dos veces al día en horas de la mañana y de la tarde, agua fue suministrada a voluntad, las sobras de ración fueron recolectadas diariamente para establecer el consumo por diferencia entre el suministro y las sobras. Las heces fueron dispuestas en un compost. Las variables zootécnicas estudiadas fueron consumo diario de alimento $(\mathrm{g})$, producción diaria de huevos (\%), peso de los huevos (g), conversión alimenticia ( $\mathrm{kg}$ de ración / docena de 
huevos y $\mathrm{kg}$ de ración $/ \mathrm{kg}$ de masa de huevo). Las características del huevo evaluadas fueron grosor de la cascara, peso específico y Unidades Haugh. Los resultados fueron sometidos a análisis de varianza y de regresión procesados en el programa SANEST (Zonta y Machado, 1996).
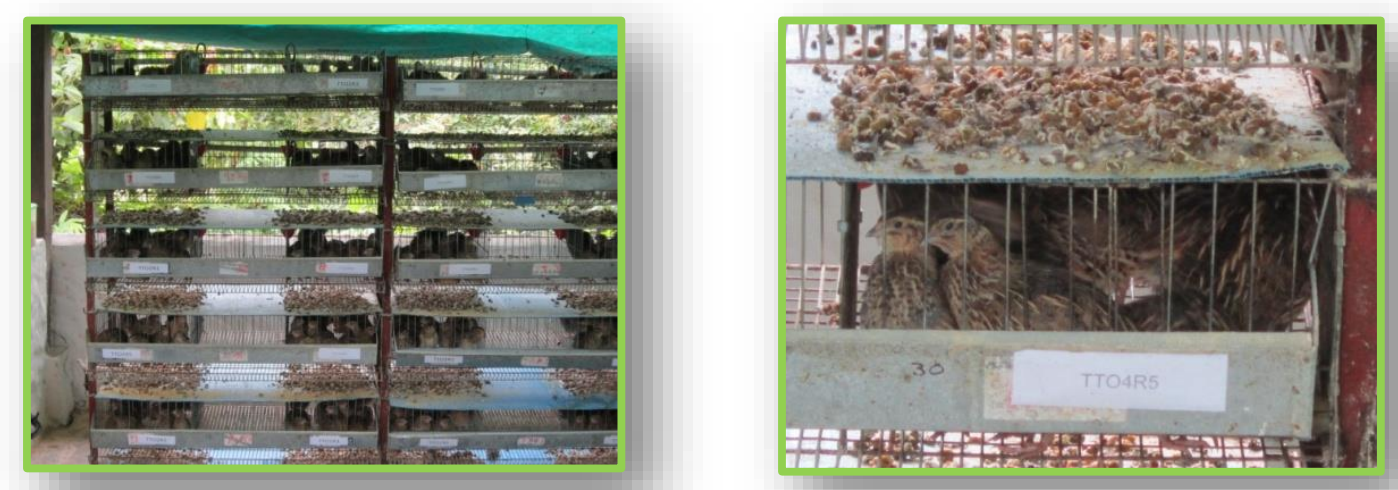

Figura 1. Betería jaula alojamiento

\section{RESULTADOS}

En la Tabla 2 se muestran los resultados de los parámetros zootécnicos obtenidos en codornices durante los primeros 114 días de vida y alimentadas con dietas conteniendo diferentes niveles de inclusión de soya integral tostada. Los resultados de los parámetros zootécnicos no presentaron diferencias significativas ( $p>0.05$ ). El mayor consumo de ración diario se obtuvo con el tratamiento con $20 \%$ de inclusión seguido en forma descendente de los tratamientos con 0, 10, 5 y $15 \%$ de grano de soya integral tostada en la ración. La producción de huevos fue mayor en el tratamiento testigo sin adición de grano de soya integral, los menores porcentajes se presentaron para las dietas con $10 \%$ y $20 \%$ de adición de grano de soya tostada, los valores intermediarios se obtuvieron con los tratamientos con $15 \%$ y $5 \%$ de inclusión de grano de soya integral tostado.

Los huevos más pesados los proporcionó el tratamiento con $5 \%$ de grano de soya integral tostado, conseguido de los tratamientos con 0, 10, 15 y 20\% de inclusión de grano de soya integral. La conversión alimenticia kg de ración/docena de 
huevos de mejor resultado fue de $4.84 \mathrm{~g}$ de ración por docena de huevo producido encontrado en los tratamientos con 0 y $15 \%$ de inclusión de grano de soya integral tostado, seguido en orden ascendente por los tratamientos con 5,10 y $20 \%$ de inclusión de grano de soya.

Tabla 2. Resultados del desempeño productivo de codornices japonesas alimentadas con diferentes niveles de inclusión de grano de soya integral tostada en la fase inicial de postura

\begin{tabular}{lccccc}
\hline \multirow{2}{*}{ Característica * } & \multicolumn{6}{c}{ Niveles de grano soya integral tostado, \% } \\
& $\mathbf{0}$ & $\mathbf{5}$ & $\mathbf{1 0}$ & $\mathbf{1 5}$ & $\mathbf{2 0}$ \\
\hline Producción huevos, \% & 60.0 & 58.0 & 54.0 & 57.0 & 54.0 \\
Peso huevo (g) & 10.5 & 10.7 & 10.5 & 10.4 & 10.4 \\
Consumo ración (g) & 24.2 & 23.8 & 23.9 & 23.0 & 24.9 \\
$\begin{array}{l}\text { Conversión Alimenticia } \\
\text { (kg ración/docena }\end{array}$ & 0.484 & 0.492 & 0.531 & 0.484 & 0.453 \\
huevos) & & & & & \\
$\begin{array}{l}\text { Conversión alimenticia } \\
\text { (kg ración/kg de huevo) }\end{array}$ & 3.84 & 3.83 & 4.21 & 3.88 & 4.43 \\
\hline No hubo diferencias significativas $(\mathrm{p}>0.05)$ & & & & \\
\hline
\end{tabular}

* No hubo diferencias significativas $(p>0.05)$

La conversión alimenticia $\mathrm{kg}$ de ración/kg de huevo producido, el tratamiento que menos cantidad de alimento, $3.83 \mathrm{~kg}$ de ración para producir un $\mathrm{kg}$ de huevo se encontró con el tratamiento con $5 \%$ de inclusión de grano de soya integral, seguido de los tratamientos con $0,15,10$ y $20 \%$ de inclusión de soya que necesitaron $3.84,3.88,4.21$ y $4.43 \mathrm{~kg}$ de ración respectivamente.

Calidad del huevo. No se presentaron diferencias significativas $(P>0.05)$ para esta variable. Los valores del grosor de la cáscara fueron similares $(0,17 \mathrm{~mm})$ para los tratamientos con 0, 10 y 20 de grano de soya (Tabla 3), siendo la cáscara más delgada para el tratamiento con el $5 \%$ de soya integral tostada, el tratamiento con mayor grosor de cáscara $(0.18 \mathrm{~mm})$, se obtuvo con el tratamiento con $15 \%$ de SIT. Los tratamientos con 5 y $20 \%$ de grano de soya presentaron igual gravedad 
específica (1.071), el menor valor se obtuvo con $15 \%$ de SIT (1.070) y el mayor con $10 \%$ de grano de soya (1.073).

Tabla 3. Resultados de la calidad del huevo y de la cáscara de codornices japonesa alimentadas con diferentes niveles de inclusión de grano de soya integral tostada en la fase inicial de postura

\begin{tabular}{lccccc}
\hline \multirow{2}{*}{ Variable * } & \multicolumn{6}{c}{ Niveles de grano soya integral tostado, \% } \\
& \multicolumn{1}{c}{$\mathbf{0}$} & $\mathbf{5}$ & $\mathbf{1 0}$ & \multicolumn{1}{c}{$\mathbf{1 5}$} & \multicolumn{1}{c}{$\mathbf{2 0}$} \\
\hline Grosor de la cascara, mm & 0.17 & 0.16 & 0.17 & 0.18 & 0.17 \\
Peso específico & 1072 & 1071 & 1073 & 1070 & 1071 \\
Unidades Haugh & 93.11 & 91.37 & 93.25 & 91.73 & 92.28 \\
\hline
\end{tabular}

${ }^{*}$ No hubo diferencias significativas $(p>0.05)$

\section{DISCUSIÓN}

El consumo diario de ración obtenido en este trabajo se encuentra próximo al consumo diario reportado por Moura et al., (2008) constataron consumo de ración de 25.2 a $27.0 \mathrm{~g}$ con dietas 2500 a $2800 \mathrm{kcal} \mathrm{EM} / \mathrm{kg}$ elaboradas con maíz, torta de soya y mogolla de trigo para codornices entre 76 y 160 días. Freitas et al., (2005) alimentando codornices de 42 días de edad y durante 168 días, con dietas conteniendo maíz y torta de soya y diferentes niveles de proteína bruta obtuvieron un consumo diario de ración de 23.98 - 24.3.1.

El relativo bajo consumo diario de ración se puede explicar por las condiciones de alta temperatura de la región donde se realizó el experimento. Los resultados de consumo de raciones en los trabajos mencionados, se explican por los niveles de energía contenidos en la ración, las raciones de mayor contenido energético presentan menor consumo por las aves. La producción de huevos no fue influenciada por los niveles de inclusión de grano de soya integral tostado en la dieta de las codornices en fase inicial de postura. Los resultados obtenidos son inferiores a los reportados por Sucupira et al., (2007) que constataron mayor producción de huevos trabajando con codornices de 84 a 147 días de edad, 
utilizando levadura de caña utilizando como fuente proteica la torta de soya. Del mismo modo, Barreto et al., (2007) obtuvieron valores mayores de postura con codornices de 56 a 168 días de edad, utilizando raciones elaboradas con maíz y torta de soya.

Estos valores discrepantes con los autores citados se explica por el hecho que los mismos iniciaron la fase experimental en el momento en que las aves estabilizaron la producción de huevos, entre tanto las aves alimentadas con inclusión de grano integral de soya tostado la fase experimental fue fijada con la edad de las aves, lo que determina los bajos valores de producción de huevos en las primeras semanas del periodo inicial de postura, lo cual afecta el promedio general de los tratamientos durante toda la fase experimental.

Los niveles de inclusión de grano soya integral tostado en la ración para codornices en postura no presentaron diferencias significativas $(p>0.05)$ para la variable peso del huevo. El peso del huevo obtenido en este trabajo supera el peso del huevo reportado por Texeira et al., (2009), de $10.2 \mathrm{~g}$ con codornices alimentadas durante dos meses con raciones elaboradas con maíz, torta de soya y soya semi-integral con $10 \%$ de extracto etéreo. Por otro lado, Costa et al., (2008) obtuvieron mayor peso del huevo con codornices alimentadas con raciones utilizando como fuente proteica la torta de soya. Los resultados del peso del huevo se explican por el contenido de nutrientes de las raciones ofrecidas que atienden los requerimientos para la fase de postura.

Los datos de conversión alimenticia asociados a $\mathrm{kg}$ de ración/docena de huevos son inferiores a los valores encontrados por Belo et al., (2000) y por Ribeiro et al., (2003). La conversión alimenticia referente al consumo de ración y la masa de huevo ( $\mathrm{kg}$ de ración/kg de huevos), la menor cantidad de alimento para producir un $\mathrm{kg}$ de masa de huevo fue expresada con la inclusión de $5 \%$ de grano soya integral tostado, valor inferior al constatado por Belo et al., (2000) y por Costa et al., (2009). Belo et al., (2000) encontraron grosor de la cáscara similar 0.16 - 0.17 
$\mathrm{mm}$, los datos se explican por el suministro adecuado de calcio en la dieta, componente esencial de la estructura de la cáscara.

Los valores de peso específico encontrados en este trabajo son próximos a encontrados por Mori et al., (2005) de 1.072 - 1.74, trabajando con codornices de 42 días de edad y una fase experimental de 168 días, alimentando las codornices con dietas con maíz y torta de soya como fuentes energética y proteica respectivamente. Costa et al., (2009) constataron peso específico de 1.068 - 1.076 de huevos de codornices japonesas durante el periodo de 74 - 94 días de edad, alimentadas con raciones con maíz y torta de soya como componentes principales.

Los valores de Unidades Haugh constatados en este trabajo, fueron superiores a los encontrados por Belo et al., 2000. Entretanto Gamboa et al., (2005) encontraron 90.73 - 95-91\% de Unidades con diferentes niveles da grano de soya integral cocido. Esta variable de calidad del huevo se explica principalmente por las condiciones y el tiempo de almacenamiento del huevo.

\section{CONCLUSIONES}

El grano de soya integral tostado puede ser incluido hasta en $20 \%$ en raciones para codornices japonesas en la fase inicial de postura, sin afectar el rendimiento zootécnico ni las características de calidad del huevo.

El grano de soya integral tostado puede constituirse en una alternativa viable de incorporación a las raciones para codornices en las zonas de producción de la leguminosa, ya que en épocas de cosecha el valor de comercialización de este producto tiende a disminuir de costo y como es un producto de excelente calidad nutricional, puede ser tostado y utilizado en explotaciones de codornices de pequeño y mediano porte. 


\section{BIBLIOGRAFÍA}

1. Barreto, S. L. T., Quirino, B. J. S., Brito, C. O., Umigi, R. T., Araujo, M. S., Coimbra, J. S. R., Rojas, E. E. G., Freitas, J. F., Reis, R. S. Níveis de energia metabolizável para codornas japonesas na fase inicial de postura. R. Bras. Zootec., 36 (1): 79-85. 2007.

2. Barreto, S. L. T., Moura, W. C. O., Reis, R. S., Hosoda, L. R., Maia, G. V. C., Pena, G. M. Soja integral processada em dietas para codornas japonesas em postura. R. Bras. Zootec., 39 (9): 1978-1983. 2010.

3. Belo, M. T. S.; Cotta, J. T. B.; Gomes, O. A. I. Niveís de energia metabolizável em rações de codornas (Coturnix coturnix japónica) na fase inicial de postura. R. Ciência e Agrotec., 24 (3): 782-793. 2000.

4. Butolo, J. E. Qualidade de ingredientes na alimentação animal. Ed Agros Comunicação, Campinas, 430 p. 2002.

5. Costa, F. G. P., Rodrigues, V. P., Goulart, C. C., Neto, R. C. L., Souza, J. G., Silva, J. H. V. Exigências de lisina digestível para codornas japonesas na fase de postura. R. Bras. Zootec., 37 (12): 2136-2140. 2008.

6. Costa, F. G. P., Rodrigues, V. P., Goulart, C. C., Vargas Jr, J. G., Silva, J. H. V., Souza, J. G. Nutritional requirements of digestible methionine + cystine for Japanese quails in production phase, R. Bras. Zootec., 38 (12): 2389-2393. 2009.

7. Freitas, A. C., Fuentes, M. F. F., Rodrigues Freitas, E. R., Sucupira, F. S., Oliveira, B. C. M. Efeito de Níveis de Proteína Bruta e de Energia Metabolizável na Dieta sobre o Desempenho de Codornas. R. Bras. Zootec., 34 (4): 838-846. 2005.

8. IDEAM. Promedios de condiciones ambientales de la Estación meteorológica. 12 p. 2002.

9. Gamboa. O. F., Díaz, J. C., Hurtado, N. V. L., Garzón, V. Efecto de los niveles de grano de soya integral cocido sobre el desempeño zootécnico y la calidad del huevo en codornices (Coturnix coturnix japonica). R. Orinoquia, 9 (2): 1521. 2005.

10. Móri, C., Garcia, E. A., Pavan, A. C., Piccinin, A., Scherer, M. R., Pizzolante, C. C. Desempenho e qualidade dos ovos de codornas de quatro grupos genéticos. R. Bras. Zootec., 34 (3): 864-869. 2005.

11. Moura, G. S., Barreto, S. L. T., Donzele, J. L., Hosoda, L. R., Pena, G. M., Angelini, M. S. Dietas de diferentes densidades energéticas mantendo constante a relação energia metabolizável: nutrientes para codornas japonesas em postura R. Bras. Zootec., 37 (9): 1628-1633. 2008.

12. National Research Council (NRC). Nutrient Requirements of poultry. 9 ed. National Academy Press, Washington, p.44-454. 1994.

13. Ribeiro, M. L. G., Silva, J. H. V., Dantas, M. O., Costa, F. G. P., Oliveira, S. F., Jordão Filho, J., Silva, E. L. Exigências Nutricionais de Lisina para Codornas durante a Fase de Postura, em Função do Nível de Proteína da Ração R. Bras. Zootec., 32 (1): 156-161. 2003. 
14. Rostagno, H. S. Albino, L. F. T., Donzele, J. L., Gomes, P. C., Oliveira, R. F., Lopes, D. C., Ferreira, A. S., Barreto, S. L. T. Tabelas Brasileiras para aves e suínos. Composição de alimentos e exigências nutricionais. $2^{a}$ ed. Viçosa: UFV, 186 p. 2005.

15. Sucupira, F. S., Fuentes, M. F. F., FREITS, E. R., BRAZ, N. M. Alimentação de codornas de postura com rações contendo levedura de cana de açúcar. Ciência Rural, 37 (2): 528-532. 2007.

16. Teixeira, R. S. C., Cardoso, W. M., Siqueira, A. A., Nogueira, G. C., Campello, C. C., Buxadé, C. C. Aspectos produtivos e qualidade de ovos de codornas japonesas submetidas a diferentes métodos de muda forçada. Ciência Anim. Bras., 10 (3): 679-688. 2009.

17. Vásquez, R. R. E., Ballesteros, C. H. H. La cría de codornices. Ed, Produmedios, Bogotá, 67 p. 2007.

18. Wineland M. Control de calidad de la cáscara mediante la determinación del peso específico de los huevos fértiles. Inf. Ganadera Ray del Pino. USA. 5 p. 1998.

19.Zonta, E. P.; Machado, A. A.; Silveira, J. P. Sistema de análise estatística para microcomputadores (SANEST). Pelotas: UFPEL, Departamento de matemáticas e estatística, 151 p. 1996. 